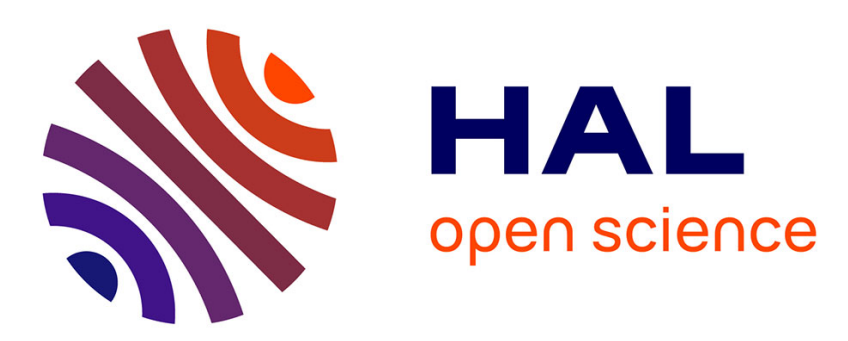

\title{
Cuve pour l'étude des spectres d'absorption infrarouge de l'hydrogène et des hydracides pour de très hautes pressions de gaz perturbateurs
}

\author{
S. Dossou, D. Clermontel, P. Boissinot, M. Bouquet, J.P. Michel, H. Vu
}

\section{- To cite this version:}

S. Dossou, D. Clermontel, P. Boissinot, M. Bouquet, J.P. Michel, et al.. Cuve pour l'étude des spectres d'absorption infrarouge de l'hydrogène et des hydracides pour de très hautes pressions de gaz perturbateurs. Revue de Physique Appliquée, 1985, 20 (11), pp.801-804. 10.1051/rphysap:019850020011080100 . jpa-00245395

\section{HAL Id: jpa-00245395 \\ https://hal.science/jpa-00245395}

Submitted on 1 Jan 1985

HAL is a multi-disciplinary open access archive for the deposit and dissemination of scientific research documents, whether they are published or not. The documents may come from teaching and research institutions in France or abroad, or from public or private research centers.
L'archive ouverte pluridisciplinaire HAL, est destinée au dépôt et à la diffusion de documents scientifiques de niveau recherche, publiés ou non, émanant des établissements d'enseignement et de recherche français ou étrangers, des laboratoires publics ou privés. 


\title{
Cuve pour l'étude des spectres d'absorption infrarouge de l'hydrogène et des hydracides pour de très hautes pressions de gaz perturbateurs
}

\author{
S. Dossou, D. Clermontel, P. Boissinot, M. Bouquet, J. P. Michel et H. Vu. \\ Laboratoire des Interactions Moléculaires et des Hautes Pressions, C.N.R.S., Université Paris-Nord, \\ 93430 Villetaneuse, France
}

(Reçu le 27 mars 1985, révisé le 28 juin, accepté le 16 juillet 1985)

\begin{abstract}
Résumé. - Nous décrivons ici un nouveau type de cuve optique pour des études d'absorption en infrarouge proche sous pression jusqu'à $10 \mathrm{kbar}$. La conception d'une cuve dont le " cœur " peut être changé suivant le type de mélanges utilisés a permis d'étudier des molécules solutées aussi différentes que l'hydrogène et les hydracides. A titre d'exemple nous donnons ici un ensemble de spectres d'absorption induite d'hydrogène dans l'azote jusqu'à $8 \mathrm{kbar}$ à température ambiante.

Abstract. - A new optical cell designed for near infrared absorption studies under pressure up to 8-10 kbar has been built. It is a double shell cell, the inner shell can be removed and replaced by another one if necessary, allowing the study of molecules as different as hydrogen and hydracids. We give here as an example the infrared absorption induced spectra of $\mathrm{H}_{2}$ in mixture with nitrogen up to $8 \mathrm{kbar}$ at room temperature.
\end{abstract}

\section{Introduction.}

Nous décrivons ici un nouveau type de cuve optique haute pression pour des études d'absorption en infrarouge sous pression jusqu'à 10 kbar. Le système de montage des fenêtres et des joints d'étanchéité est complètement différent de celui utilisé dans les cuves conventionnelles, et présente par rapport à celui-ci certains avantages qui seront détaillés plus loin.

A l'origine de cette conception, il y a eu tout d'abord un besoin tout à fait spécifique à nos études spectroscopiques de certains gaz sous haute pression. Nous sommes appelés en effet à étudier des molécules très variées telles que $\mathrm{H}_{2}, \mathrm{CO}$, hydracides, etc... dont le comportement chimique est très différent vis-à-vis du matériau du corps de la cuve. A titre d'exemple, on s'est aperçu que l'alliage APX4 résiste très bien à $8 \mathrm{kbar}$ et $300 \mathrm{~K}$, à la corrosion par les hydracides, mais par contre est fortement fragilisé par l'hydrogène au-delà de $3 \mathrm{kbar}$. Le $\mathrm{BeCu}$, par contre dans les mêmes conditions de pression et de température, résiste très bien à l'action de $\mathrm{H}_{2}$, mais moins bien à celle des hydracides. Le besoin s'est donc fait sentir d'avoir un corps de cuve approprié, d'une part à chaque type de gaz étudié, et d'autre part au domaine de pression et de température exploré dans l'étude, d'où l'idée d'avoir une cuve à double corps dont le " cœur " interne peut facilement être changé. Nous avons donc été amenés à concevoir un système répondant aux impératifs suivants :

a) supporter des pressions allant jusqu'à $10 \mathrm{kbar}$ en phase gazeuse dans un domaine de température compris entre 77 et $300 \mathrm{~K}$;

b) " cours" internes facilement interchangeables;

c) ensemble devant être, autant que possible, de réalisation simple et de dimensions réduites afin de pouvoir être très maniable.

\section{Description de la cuve réalisée.}

La figure 1 montre le système qui a été adopté et qui se compose d'une enveloppe externe contenant un " cour " interne constituant la cuve proprement dite. Le " cœur ", soit en APX4, traité à 90 hbar, soit en $\mathrm{BeCu}$ traité à $100 \mathrm{hbar}$, est un simple cylindre de $20 \mathrm{~mm}$ de long, $20 \mathrm{~mm}$ de diamètre et $4 \mathrm{~mm}$ d'alésage interne. Les deux faces planes sont rectifiées et bien parallèles entre elles. Deux fenêtres en saphir (cylindres de $10 \mathrm{~mm}$ de diamètre et $10 \mathrm{~mm}$ d'épaisseur) à faces parallèles avec planéité optique, sont appliquée aux deux extrémités du cylindre. L'enveloppe, cylindre en APX4 traité à $90 \mathrm{hbar}$, a simplement le rôle d'assurer, d'une part l'étanchéité par serrage des deux fenêtres en saphir contre le corps de cuve, et d'autre part le serrage de l'arrivée de pression qui s'effectue par l'intermédiaire d'un trou de $1 \mathrm{~mm}$ de diamètre pratiqué au centre du cylindre. 


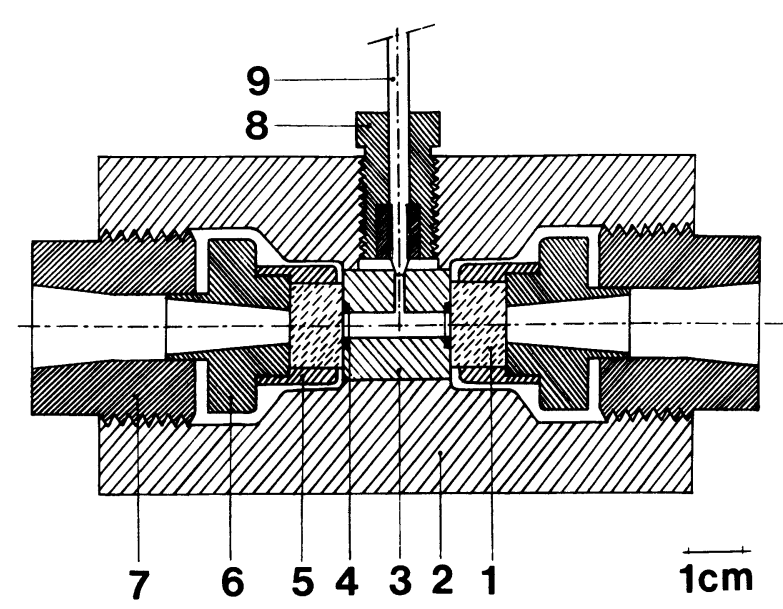

Fig. 1. - Schéma de la cuve haute pression.

1. fenêtre en saphir

2. corps externe en APX4

3. corps interne en $\mathrm{BeCu}$ ou en APX4

4. système de joints haute pression

5. boîtier de fenêtre en laiton

6. porte-fenêtre en APX4

7. bouchon en $\mathrm{BeCu}$

8. bouchon en $\mathrm{BeCu}$

9. tube haute pression

[Schematic representation of the high pressure cell

1. Sapphire window

2. Outer APX4 shell

3. Inner $\mathrm{BeCu}$ or $\mathrm{APX} 4$ shell

4. Packing assembly

5. Window cap

6. Window plug

7. $\mathrm{BeCu}$ sleeve-nut

8. $\mathrm{BeCu}$ hexagonal nut

9. High pressure entry.]

Toute la difficulté réside dans le système assurant l'étanchéité entre fenêtres et cuve, depuis un vide primaire jusqu'à une pression de $10 \mathrm{kbar}$. Dans le montage classique des cuves conventionnelles les fenêtres sont montées suivant le principe de Poulter [1], c'est-à-dire, reposent chacune sur une embase plane avec contact étanche sur plan optique. L'embase est ensuite montée sur une extrémité de la cuve avec étanchéité par un système de joints haute pression, en général du type « en $T$ » [2] ou du type « champignon » [3].

Dans le présent ensemble nous adoptons un système de montage optique complètement différent qui a l'avantage d'être particulièrement simple, tant dans la réalisation, que dans l'utilisation au montage et au démontage. Les fenêtres en saphir sont plaquées directement contre le corps de cuve (Fig. 1) et l'étanchéité est assurée non plus par planéité optique mais par le système de joints décrit ci-dessous. Aux deux extrémités rectifiées de la cuve interne sont pratiqués deux logements de joints profilés comme le montre la figure 2. Chaque logement contient le système de joints

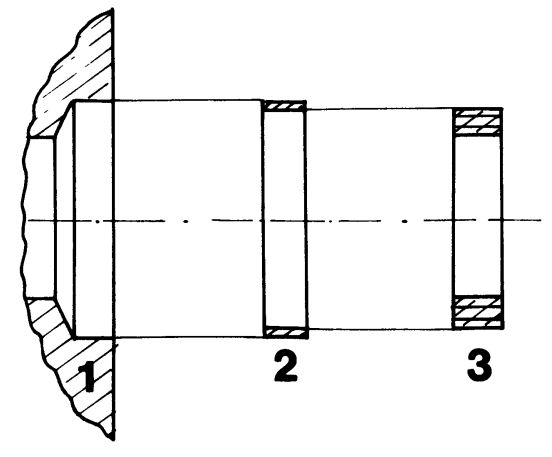

$0,5 \mathrm{~cm}$

Fig. 2. - Corps interne et système de joints de la cuve.

1. corps interne

2. bague anti-extrusion en laiton

3. joint en téflon

[Inner shell and packing assembly

1. Inner shell

2. Anti-extrusion brass ring

3. Teflon packing.]

constitué, à l'extérieur, d'une bague anti-extrusion en laiton (diamètres 7,7 et $7 \mathrm{~mm}$, épaisseur $1,3 \mathrm{~mm}$ ), et à l'intérieur, d'un joint plat en téflon (diamètres 7 et $4 \mathrm{~mm}$, épaisseur $1,6 \mathrm{~mm}$ ) monté serré dans la bague en laiton, elle-même ajustée dans le corps. Au montage, le système de joints dépasse le logement prévu, mais le serrage par les écrous (7) (voir Fig. 1) les met en place définitivement. L'expérience a montré qu'un tel système de joints est réutilisable après plusieurs montages et démontages. Le rôle de la bàgue antiextrusion est essentiel. Sans elle le joint en téflon ne peut résister au-delà de 3 à $4 \mathrm{kbar}$. On pourrait également si nécessaire, lui donner un profil triangulaire pour rendre le système encore plus autoserreur. A la place des joints plats en téflon, des joints toriques en élastomère disponibles dans le commerce ont été également testés avec succès (Viton, etc...). Le serrage des fenêtres par les écrous (7) pourrait paraître à première vue critique car il faut un serrage suffisant pour équilibrer la poussée de la pression sur les fenêtres. En fait, dans la pratique il suffit de serrer modérément au départ, de façon à amener en contact fenêtres et extrémités de la cuve. La bague anti-extrusion en laiton et le joint en téflon permettent de rattrapper les jeux dûs à la poussée de la pression.

L'ensemble de la cuve a été testé à température ambiante jusqu'à $9 \mathrm{kbar}$ et travaille régulièrement jusqu'à 8 kbar en gaz. Cette limite de pression n'est pas celle imposée par la cuve, mais celle de l'installation haute pression gazeuse dont nous disposons actuellement (compresseur, valves et manomètres). Il est très probable que le système cuve-fenêtres-joints peut fonctionner à plus haute pression encore. D'autres 
systèmes de joints sont également envisageables et nous projetons de tester l'efficacité du système au-delà de $10 \mathrm{kbar}$. Ce nouveau système, imaginé pour les besoins d'une étude très spécialisée, s'est révélé d'une utilité plus générale.

\section{Avantages et inconvénients de la cuve réalisée.}

Nous allons dans ce qui suit discuter des avantages et des inconvénients de ce nouveau système de montage des fenêtres par rapport au système conventionnel.

Le système conventionnel de montage pour haute pression comprend en fait deux types de montage :

- pour des pressions relativement faibles (inférieures à $2 \mathrm{kbar}$ ), on peut assurer l'étanchéité entre la fenêtre et le corps de cuve, grâce à un système utilisant un joint souple (argent, cuivre ou téflon) que l'on écrase entre la fenêtre et le corps de cuve $[4,5]$. C'est le système le plus simple à réaliser et le meilleur marché que l'on puisse imaginer. Sa simplicité permet aussi de très faibles dimensions de cuve, mais son efficacité devient aléatoire au-dessus de 2 kbar à cause de l'extrusion du joint;

- pour des pressions supérieures à 2 kbar on est obligé d'utiliser un système de joint plus complexe, logé et autoserreur du type à aire non supportée $[6,7,8]$ où, d'une part le joint est « logé » (extrusion fortement réduite), et d'autre part l'effort de serrage sur le joint d'étanchéité est proportionnel à la pression que celui-ci doit supporter. Nous avons utilisé jusqu'à présent ce système dans nos cuves pour $10 \mathrm{kbar}$ et il s'est avéré efficace, mais c'est un système nettement plus sophistiqué que le premier et qui exige un soin d'exécution et une précision d'usinage qui en augmente considérablement le prix. Par ailleurs, l'encombrement de ce système complexe de joints, impose des dimensions importantes à la cuve.

Le système que nous proposons est intermédiaire entre les deux systèmes conventionnels que nous venons d'exposer, alliant, tout au moins partiellement, la simplicité du premier à l'efficacité du second. En effet, ce système a la simplicité d'un système de joint " écrasé " et "logé », tout en ayant l'efficacité des joints autoserreurs. Son encombrement est nettement plus réduit et permet des dimensions de cuve moins importantes.

Etant donné les dimensions des fenêtres utilisées ( $10 \mathrm{~mm}$ de diamètre) et du joint choisi, l'effort à $10 \mathrm{kbar}$ sur l'écrou de serrage n'est que de 4 tonnes. Dans un système conventionnel utilisant par exemple un joint " champignon " avec des fenêtres de $10 \mathrm{~mm}$ de diamètre, il faudrait des embases de $12 \mathrm{~mm}$ de diamètre et un système de joints d'embase de 16 à $20 \mathrm{~mm}$ de diamètre, on aurait alors pour $10 \mathrm{kbar}$ un effort de 20 à 30 tonnes environ sur l'écrou de serrage.

Le nouveau système présente donc, outre la simplicité de réalisation, l'avantage de réduire considérablement l'effort sur l'écrou de serrage des fenêtres et par suite les dimensions globales de la cuve, ce qui peut être très utile dans certain cas, particulièrement en spectroscopie où l'on a intérêt à miniaturiser les cuves.

Par ailleurs, il est à remarquer, que non seulement les dimensions globales de la cuve ont pu être réduites par rapport au système conventionnel, mais que le volume de travail a pu être fortement diminué $\left(240 \mathrm{~mm}^{3}\right.$ au lieu de quelques $\mathrm{cm}^{3}$ ).

L'inconvénient du présent montage réside certainement dans la difficulté de trouver aux très basses températures des joints qui ne perdent pas leur élasticité. Il n'est cependant pas impossible d'imaginer un système de joints plus complexe, métallo-plastiques, alliant à la fois la résistance mécanique du métal et la souplesse du plastique à basse température. Cela devrait permettre de réaliser le préserrage et l'étanchéité préliminaire pour que le système de joints fonctionne en autoserreur à haute pression. La mise au point est en cours, d'une part pour tester l'efficacité de ce type de montage aux pressions plus élevées (supérieures à $10 \mathrm{kbar}$ ), et d'autre part pour son adaptation à l'utilisation aux basses températures.

Par ailleurs, nous voulons signaler un inconvénient inhérent à une miniaturisation trop poussée d'une cuve optique, c'est-à-dire d'un trop faible volume de travail : le rapport du volume de travail au volume mort du circuit haute pression étant réduit, cela peut introduire une imprécision sur la mesure de la quantité de gaz introduite dans la cuve. De plus, dans un si

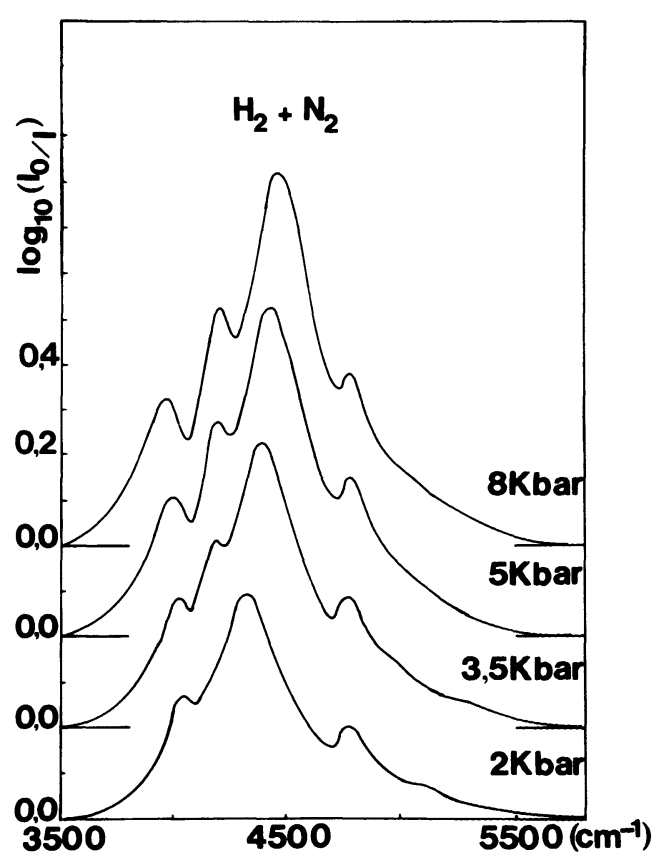

Fig. 3. - Spectres d'absorption induite dans le proche infrarouge de $\mathrm{H}_{2}$ dans l'azote jusqu'à 8 kbar à température ambiante.

[Induced absorption spectra in the near infrared region of $\mathrm{H}_{2}$ in nitrogen up to $8 \mathrm{kbar}$ at $300 \mathrm{~K}$.] 
petit volume utile, toute diminution du nombre des molécules actives par suite de phénomènes parasites tels que corrosion, adsorption, fuites, etc..., prend une proportion gênante.

\section{Résultats. Conclusion.}

Nous donnons figure 3, un exemple de spectres d'absorption induite par la pression dans l'infrarouge proche, obtenus avec $\mathrm{H}_{2}$ comprimé par l'azote jusqu'à $8 \mathrm{kbar}$ à $300 \mathrm{~K}$. Le spectromètre utilisé est un Perkin Elmer $112 \mathrm{C}$ à simple faisceau, transformé pour l'utilisation des cuves haute pression, muni d'un prisme en $\mathrm{LiF}$ et d'un détecteur thermocouple à fenêtre en $\mathrm{KBr}$ (résolution $5 \mathrm{~cm}^{-1}$ dans le cas présent).

Les autres résultats des études qui ont été faites avec cette cuve feront l'objet de publications à part.

En conclusion, nous avons réalisé une cuve optique pour haute pression en gaz, alliant l'efficacité à la maniabilité lors du montage et du démontage. La technique du corps double nous a permis d'adapter le matériau constituant le " cour » à la nature des gaz étudiés. Nous envisageons de prolonger ces études à plus haute pression.

\section{Bibliographie}

[1] Poulter, T. C. and Benz, C., Phys. Rev. 40 (1932) 860, 872.

[2] Coulon, R., J. Rech. C.N.R.S. 9 (1958) 305.

[3] Jean-Louis, M. et Vu, H., Revue Phys. Appl. 7 (1972) 89.

[4] Robin, S., J. Chim. Phys. 48 (1951) 145.

[5] Marteau, Ph., Vu, H. et Vodar, B., Revue Phys. Appl. 4 (1969) 463.
[6] Bridgman, P. W., The Physics of High Pressure (Ed. Bell G. and Sons, London) 1958, p. 34.

[7] TsikuIs, D. S., Handbook of Techniques in High Pressure Research and Engineering (Ed. Plenum Press, New York) 1968, p. 225.

[8] Clermontel, D., Michel, J. P., Khatibi, P. and Vu, H., Infrared Phys. 18 (1978) 229. 